\title{
Impact of non-selective beta blockers on portal hypertension and hepatic elasticity in hepatitis $C$ virus-related liver cirrhosis
}

\author{
Sahar G. Zaghloul ${ }^{1}$, Essam A. Wahab ${ }^{1}$, Waseem M. Seleem ${ }^{1}$, Amr S. Hanafy, ${ }^{1, *}$, \\ Ahmed Fathy Gomaa ${ }^{1}$, Kh Lakouz ${ }^{2}$, A.I. Amin ${ }^{3}$ \\ ${ }^{1}$ Hepato-gastroenterology Unit, Department of Internal Medicine, Zagazig University, Zagazig, Egypt; \\ ${ }^{2}$ Department of Diagnostic Radiology, Faculty of Medicine, Zagazig University, Zagazig, Egypt; \\ ${ }^{3}$ Hepato-gastroenterology Unit, Department of Internal Medicine, Faculty of Medicine, Port Said University, Zagazig, Egypt.
}

\begin{abstract}
Summary Portal hypertension and its complications are the leading causes of morbidity and mortality in patients with liver cirrhosis. Noninvasive assessment of liver stiffness had been an effective tool for assessment of fibrosis progression in chronic liver disease. It was intended to assess liver stiffness measurement (LSM), portal vein diameter (PVD), splenic bipolar diameter (SD), and the platelet count/spleen diameter (PC/SD) ratio in patients who test positive for the hepatitis $\mathrm{C}$ virus (HCV) and to study the impact of non-selective beta blockers (NSBB) on the grade of esophageal varices (EVs) and liver elasticity. Subjects were 80 patients with ChildPugh grade A or B compensated cirrhosis who tested positive for HCV. All of the patients underwent a laboratory workup including AFP, HCV antibodies, HCV RNA, HBsAg, LSM according to real-time elastography, upper gastrointestinal endoscopy (UGIE) to detect and grade EVs, calculation of the PC/SD ratio, and measurement of the PVD and SD according to real-time abdominal ultrasonography. All patients were given the maximum tolerated dose of NSBB for three months, and UGIE, LSM, PC/SD, PVD, and SD were subsequently reassessed and reported. LSM and the PC/SD ratio were exceptional noninvasive tools for prediction of significant EVs (grade $\geq$ II, $p<0.001$ ) with a sensitivity $82.4 \%$ and a specificity $82.6 \%$ at a cutoff point $18 \mathrm{kPa}$ for LSM, and a sensitivity $94.1 \%$ and specificity $69.6 \%$ at a cutoff point 880 for the PC/SD ratio. LSM is highly correlated with PVD, the PC/SD ratio, SD, and the Child-Pugh score. NSBB significantly decreased PVD. The percent change in PVD significantly correlated with LSM, the grade of EVs, and SD. Findings indicated that LSM is a noninvasive, rapid, and reproducible tool with which to detect portal hypertension and EVs. NSBB therapy can effectively decrease PVD and may consequently improve the EV grade with no significant impact on LSM in patients with liver cirrhosis.
\end{abstract}

Keywords: Liver stiffness measurement, portal hypertension, esophageal varices, non-selective beta blockers

\section{Introduction}

Portal hypertension is believed to be the main trigger for most complications in patients with liver cirrhosis. A hepatic venous pressure gradient $(\mathrm{HVPG}) \geq 10$ $\mathrm{mm} \mathrm{Hg}$ is necessary for the development of ascites, esophageal varices (EVs), and all other complications

*Address correspondence to:

Dr. Amr S Hanafy, Department of Internal Medicine, Zagazig

University, Zagazig city, Egypt.

E-mail: dr_amr_hanafy@yahoo.com of liver cirrhosis (1). Clinically significant portal hypertension (CSPH) is invariably found in patients with decompensated liver disease (2); its presence is an independent predictor of clinical decompensation in $50-70 \%$ of patients (3). The presence of EVs, as a complication of CSPH, is an independent predictor of significant morbidity, so all patients with compensated cirrhosis should be screened for the presence of EVs (4).

Patients with liver cirrhosis must sometimes undergo invasive procedures to diagnose EVs and CSPH, such as liver biopsy and hepatic vein catheterization. These procedures require specific experience and carry some 
risk, so simple, noninvasive, accurate, and objective diagnostic tools need to be developed for high-risk patients. A reproducible estimation of liver stiffness (LS) according to transient hepatic elastography has been developed as a noninvasive method to diagnose CSPH and EVs in patients with compensated cirrhosis (5).

Non-selective beta-blockers (NSBB) have been used since 1981 as a therapeutic option for portal hypertension in patients with liver cirrhosis. Patients with refractory ascites experience a diminished sensitivity to the NSBB due to increased levels of splanchnic pro-inflammatory cytokines; the beneficial effects of NSBBs may decrease, and NSBBs may even be harmful (6).

One aim of the current study was to assess hepatic elasticity, portal vein diameter (PVD), the platelet count/spleen diameter (PC/SD) ratio, and spleen bipolar diameter (SD) in patients with compensated liver cirrhosis who were also infected with HCV. A second aim of this study was to examine the impact of NSBB on the grade of EVs and liver stiffness measurement (LSM).

\section{Patients and Methods}

\subsection{Study design and settings}

A case control, prospective observational study was conducted in the Hepatogastroenterology and Endoscopy units of the Department of Internal Medicine in cooperation with the Advanced Center for Liver Disease of Zagazig University Hospital, Egypt over a six-month period from November 2017 to April 2018.

\subsection{Subject population}

Potential subjects were 300 patients with liver cirrhosis and who were infected with HCV who were seen at an outpatient clinic and who were scheduled for diagnostic upper GI endoscopy. Subjects were 80 patients with compensated cirrhosis who met the inclusion criteria while not meeting the exclusion criteria (Figure 1).

\subsection{Inclusion and exclusion criteria}

Patients had Child-Pugh A or B grade compensated liver cirrhosis and tested positive for $\mathrm{HCV}$ infection. Patients infected with the hepatitis B virus or who had hepatocellular carcinoma, portal vein thrombosis, who had undergone sclerotherapy or band ligation to treat EVs, patients who had previously received or who were ineligible to receive NSBBs (obstructive airway disease, peripheral arterial disease, or brittle diabetes), patients who had Child-Pugh grade $\mathrm{C}$ cirrhosis, patients

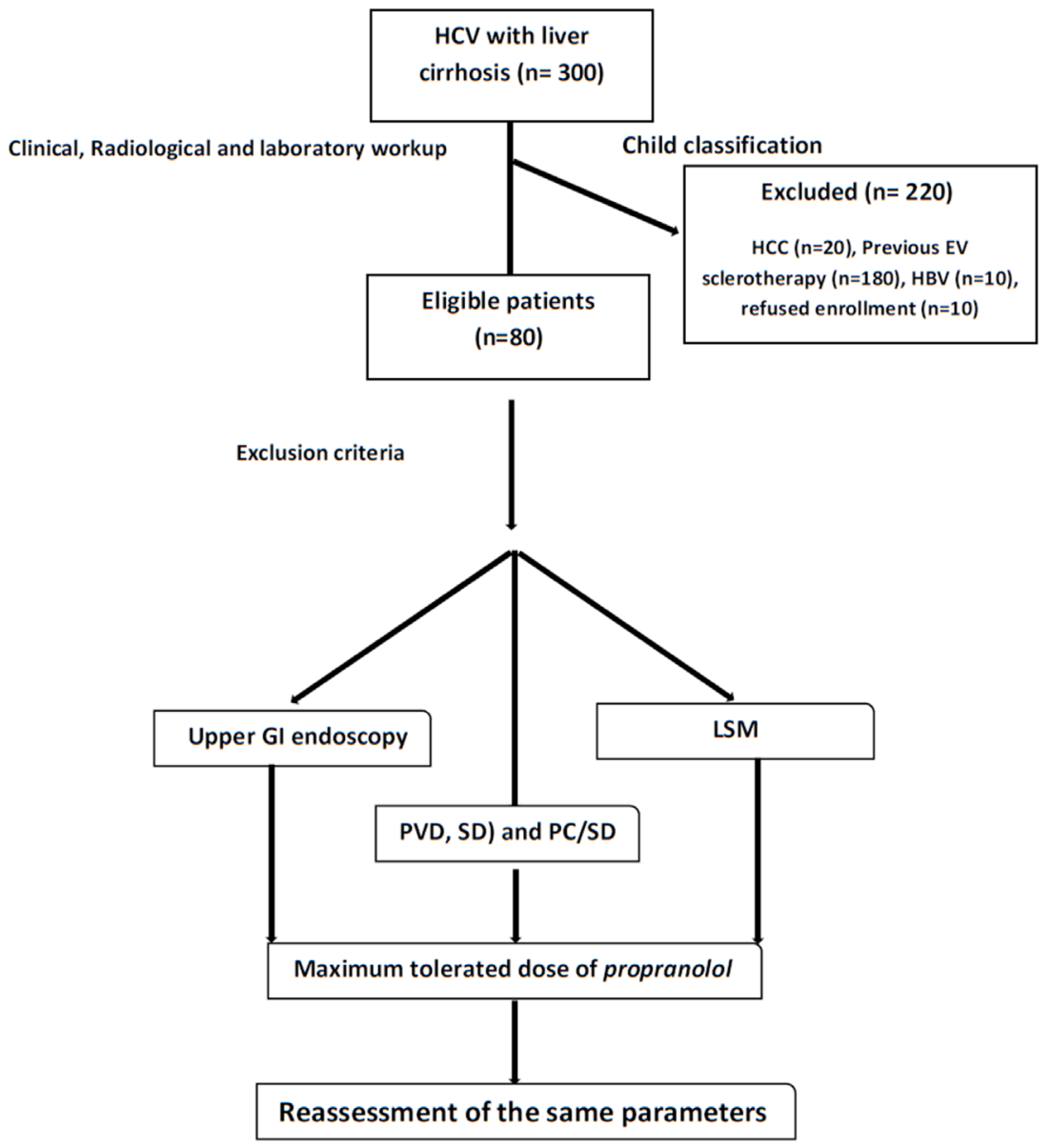

Figure 1. Flowchart for patients in this study. 
who did not have EVs, and patients who missed followup or who declined to participate in this study were excluded. All of the enrolled patients gave informed consent via a form developed by the research team. This study was approved by the ethical committee of Zagazig University. All information gathered from patients was kept confidential.

\subsection{Methods}

All patients had their history taken and underwent a physical examination and laboratory testing that included a complete blood count (CBC), platelet count, liver and kidney function tests, the international normalized ratio (INR), measurement of alpha fetoprotein (AFP), and serum markers for $\mathrm{HCV}$ and HBV. PVD and SD had been assessed using transabdominal ultrasound (Famio 5 ultrasound Machine, Abex Medical System, Toshiba, Japan). Variations in PVD during respiratory phases were addressed by measuring PVD during inspiration, expiration, and at rest. The normal PVD was less than $13 \mathrm{~mm}$ and SD was less than $130 \mathrm{~mm}$. The platelet count (PC) was divided by $\mathrm{SD}$ to yield the $\mathrm{PC} / \mathrm{SD}$ ratio. Upper gastrointestinal endoscopy (UGIE) served as a standard diagnostic modality for EVs. All patients underwent UGIE using GIF- XP160 video endoscopy (Exera 160 series, Olympus Endoscopy System, Japan). EVs were graded into the four grades of I, II, III, and IV according to the modified Paquet classification.

Estimation of liver stiffness using real-time elastography was accomplished by measuring the velocity of elastic shear waves in the liver parenchyma generated by the mechanical push (using a Philips IU22 ultrasound machine). The medium reading of the tissue elasticity was calculated and expressed in $\mathrm{kPa}$. The success rate of the examination was calculated as the ratio of the number of validated measurements made by the machine and the total number of attempted measurements during the same examination. The median value for validated measurements was used to represent liver stiffness (7).

All patients had been scheduled to receive the maximum tolerated dose of propranolol that decreases the basal heart rate by $25 \%$ but not below 60 beats per minute (5). The same patients were re-evaluated after 3 months by measuring UGIE, LSM, PVD, SD, and the $\mathrm{PC} / \mathrm{SD}$ ratio again.

\subsection{Data processing and analysis}

All calculations were performed using the computer programs SPSS (Statistical Package for the Social Science; SPSS Inc., Chicago, IL, USA version 18.0). Data were statistically described in terms of mean $\pm \mathrm{SD}$, median and range, or frequencies and percentages when available. The Mann Whitney (U) and Kruskal Wallis tests were used when appropriate. Sensitivity and specificity were used to represent the accuracy of the tests. Receiver operator characteristic (ROC) analysis was used to determine the optimum cutoff value for the diagnostic variables studied. To determine the significant independent predictors for the occurrence and the grade of significant EVs, univariate and multivariate regression models were constructed. $P$ values $<0.05$ was considered statistically significant.

\section{Results}

The mean daily dose of propranolol used in the study was $66.95 \pm 17 \mathrm{mg}$ (range: $30-80 \mathrm{mg}$ ), which caused a significant decrease in the mean heart rate from $79.05 \pm$ 9.02 (range: $60-95 \mathrm{~b} / \mathrm{m}$ ) pre- treatment to $61.15 \pm 4.73$ (range: $55-71 \mathrm{~b} / \mathrm{m})$ post-treatment $(p<0.001)$.

According to endoscopic evaluation pre-treatment, 46 patients (57\%) had grade I EVs while 32 (40\%) had grade II and only 2 patients had grade III EVs (2.5\%). Post-treatment endoscopic examination revealed that 4 patients had no EVs (5\%), 42 patients had grade I EVs (52.5\%), 32 had grade II EVs (40\%) and only 2 patients had grade III EVs (2.5\%).

There were no significant changes between pretreatment and post-treatment values for SD, LSM, and PC/SD $(p=0.5,0.77$, and 0.08), but PVD decreased significantly $(p<0.001)$ (Table 1$)$. The percent change in PVD was correlated with the percent change in EV grade, LSM, and SD ( $p=0.05,0.001$, and 0.05) (Table 2).

Pre- and post-treatment, the grade of EVs was

Table 1. Mean and median values for variables pre-treatment in the patients studied

\begin{tabular}{|c|c|c|c|c|}
\hline Variable & Pre-treatment, $(n=80)$ & Post-treatment, $(n=80)$ & Test of sig. & $p$ \\
\hline PVD: (mm) & & & $\mathrm{t}$ & $<0.001$ \\
\hline Mean \pm SD & $12.8 \pm 1.34$ & $11.44 \pm 1.93$ & 5.87 & \\
\hline Range & $10-15$ & $7-15$ & & \\
\hline Splenic diameter: $(\mathrm{cm})$ & & & $\mathrm{t}$ & 0.50 \\
\hline Mean $\pm \mathrm{SD}$ & $14.77 \pm 2.17$ & $14.8 \pm 2.18$ & 0.84 & \\
\hline Range & $7.5-18.3$ & $7.5-18$ & & \\
\hline LSM: (kpa) & & & W & 0.77 \\
\hline Median (Range) & $18.15(8-44.1)$ & $17.6(7.8-43)$ & 0.29 & \\
\hline $\mathrm{PC} / \mathrm{SD}$ ratio & & & W & \\
\hline Median (Range) & $800(188-4667)$ & $720(226-4440)$ & 1.47 & 0.08 \\
\hline
\end{tabular}


significantly correlated with PVD, SD, and LSM and inversely correlated with the PC/SD ratio (Table 3 ). In the pre-treatment evaluation, LSM at a cutoff value of $18 \mathrm{kPa}$, a PC/SD ratio of 808 , and PVD of 12.5 mm predicted EVs ( $\geq$ grade II), while LSM at a cutoff value of $16.8 \mathrm{kPa}$, a PC/SD ratio of 720 , and PVD of $11.5 \mathrm{~mm}$ post-treatment predicted $\mathrm{EVs} \geq$ grade II, thus indicating the impact of adding NSBB (Table 4). LSM was significantly correlated with both BMI and age ( $p$ $<0.05$ and $<0.001)$.

The pre- and post-treatment mean values for PVD $(12.8 \pm 1.34$ vs. $11.44 \pm 1.93 \mathrm{~mm})$ differed significantly $(p<0.001)$. PVD at a cutoff value of $12.5 \mathrm{~mm}$ had a sensitivity $82.4 \%$ and a specificity of $47.8 \%$ at predicting significant EVs. PVD was significantly correlated with age and BMI $(p=0.02$ and 0.001$)$. SD

Table 2. Correlation between the percent change in the portal vein diameter (PVD) and the percent change in the grade of esophageal varices, liver stiffness measurement, and splenic diameter in the patients studied

\begin{tabular}{llc}
\hline \multirow{2}{*}{ Variable } & \multicolumn{2}{c}{$\%$ change in PVD } \\
\cline { 2 - 3 } & $r$ & $P$ \\
\hline \% change in EV grade & 0.33 & $<0.05$ \\
\% change in LSM & 0.63 & $<0.001$ \\
\% change in splenic diameter & 0.34 & $<0.05$ \\
\hline
\end{tabular}

did not change significantly with NSBB $(14.77 \pm 2.17$ cm vs. $14.8 \pm 2.18, p=0.5)$ post-treatment. There was no significant correlation between SD and the NSBB dose $(p=0.88)$. The $\mathrm{PC} / \mathrm{SD}$ ratio was highly inversely correlated with the grade of EVs, PVD, and SD during pre- and post-treatment assessments $(p<0.001)$. The $\mathrm{PC} / \mathrm{SD}$ ratio was significantly inversely correlated with age and BMI $(p<0.001)$. LSM was highly correlated with the grade of EVs pre- and post-treatment ( $p<$ $0.001)$. LSM at a cutoff value $18 \mathrm{kPa}$ had a sensitivity $82.4 \%$ and a specificity of $82.6 \%$ at predicting EVs ( $\geq$ grade II). The NSBB dose was not significantly correlated with LSM, SD, or the PC/SD ratio but was significantly correlated with the percent change in PVD $(p<0.001)$.

\section{Discussion}

Portal hypertension and its complications are the leading causes of morbidity and mortality in patients with liver cirrhosis. The most important consequences are those that constitute decompensation of cirrhosis, such as ascites, variceal hemorrhage, and encephalopathy. The median survival of a patient without complications of portal hypertension is longer than 12 years, whereas it is shorter than 2 years for a decompensated patient (1).

NSBBs are commonly used to decrease portal

Table 3. Correlation between liver stiffness measurements, portal vein diameter, beta blocker dose, grade of esophageal varices, platelet/spleen ratio, and spleen diameter pre- and post-treatment among the patients studied

\begin{tabular}{|c|c|c|c|c|c|c|c|c|c|c|}
\hline \multirow{2}{*}{ Variable } & \multicolumn{2}{|c|}{$\operatorname{PVD}(n=80)$} & \multicolumn{2}{|c|}{ BB dose $(n=80)$} & \multicolumn{2}{|c|}{ EV grade $(n=80)$} & \multicolumn{2}{|c|}{$\operatorname{LSM}(n=80)$} & \multicolumn{2}{|c|}{$\mathrm{SD}(n=80)$} \\
\hline & $r$ & $p$ & $r$ & $p$ & $r$ & $p$ & $r$ & $p$ & $r$ & $p$ \\
\hline \multicolumn{11}{|l|}{ Pre-treatment } \\
\hline BB dose & -0.33 & 0.02 & -- & ---- & --- & ---- & -- & ---- & --- & ---- \\
\hline EV grade & 0.54 & $<0.001$ & -0.17 & & --- & ----- & --- & ---- & --- & ---- \\
\hline LSM & 0.63 & $<0.001$ & 0.04 & 0.83 & 0.53 & $<0.001$ & -- & --- & --- & --- \\
\hline $\mathrm{SD}$ & 0.31 & 0.04 & -0.05 & 0.77 & 0.62 & $<0.001$ & 0.55 & $<0.001$ & --- & ---- \\
\hline $\mathrm{PC} / \mathrm{SD}$ ratio & -0.49 & $<0.001$ & -0.11 & 0.17 & -0.71 & $<0.001$ & -0.62 & $<0.001$ & -0.79 & $<0.001$ \\
\hline \multicolumn{11}{|l|}{ Post-treatment } \\
\hline $\mathrm{BB}$ dose & -0.02 & 0.90 & & & & & & & & \\
\hline EV grade & 0.42 & 0.007 & -0.19 & 0.25 & ---- & ---- & & & & \\
\hline LSM & 0.67 & $<0.001$ & -0.05 & 0.75 & 0.49 & $<0.001$ & & & & \\
\hline $\mathrm{SD}$ & 0.25 & 0.12 & -0.03 & 0.88 & 0.28 & 0.08 & 0.61 & $<0.001$ & ---- & ---- \\
\hline $\mathrm{PC} / \mathrm{SD}$ ratio & -0.51 & $<0.001$ & -0.19 & 0.23 & -0.53 & $<0.001$ & -0.63 & $<0.001$ & -0.79 & $<0.001$ \\
\hline
\end{tabular}

Table 4. Validity of cutoff values for liver stiffness measurement, the platelet/spleen ratio, and portal vein diameter in diagnosis of esophageal varices pre- and post- treatment

\begin{tabular}{|c|c|c|c|c|c|c|}
\hline Test & Sensitivity & Specificity & PPV & NPV & Kappa & $p$ \\
\hline \multicolumn{7}{|l|}{ Pre-treatment } \\
\hline $\mathrm{LSM}>18 \mathrm{kPa}$ & 82.4 & 82.6 & 77.8 & 86.4 & 0.64 & $<0.001$ \\
\hline $\mathrm{PC} / \mathrm{SD}$ ratio $\leq 880$ & 94.1 & 69.6 & 69.6 & 94.1 & 0.61 & $<0.001$ \\
\hline $\mathrm{PV}>12.5 \mathrm{~mm}$ & 82.4 & 47.8 & 53.8 & 78.6 & 0.28 & 0.04 \\
\hline \multicolumn{7}{|l|}{ Post-treatment } \\
\hline $\mathrm{LSM}>16.8 \mathrm{kPa}$ & 90.9 & 62.5 & 52.6 & 93.8 & 0.45 & $<0.003$ \\
\hline $\mathrm{PC} / \mathrm{SD}$ ratio $\leq 720$ & 90.9 & 62.5 & 52.6 & 93.8 & 0.45 & $<0.003$ \\
\hline $\mathrm{PV}>11.5 \mathrm{~mm}$ & 72.7 & 62.5 & 47.1 & 83.3 & 0.31 & $<0.05$ \\
\hline
\end{tabular}


hypertension in patients with compensated cirrhosis in order to facilitate primary and secondary prevention of first variceal bleeding. Propranolol is the most commonly used NSBB that causes a significant reduction in portal pressure. The current study used propranolol at a dose that achieved a $25 \%$ reduction in heart rate. In patients with high-risk varices, an NSBB significantly decrease the incidence of first variceal hemorrhage.

In patients who have already bled from varices, an NSBB prevented the recurrence of variceal hemorrhage when used in combination with endoscopic variceal therapy. Recent data suggested that in patients with compensated cirrhosis (i.e., patients who have CSPH with no or small varices), NSBB provides protection from clinical decompensation (8).

In the current study, females tended to have a lower PC/SD ratio and PVD but higher LSM. These findings agreed with the results of Castera et al. (9) regarding a non-significant difference between genders. In the study by Castera et al., SD and PVD were significantly correlated with age, and the PC/SD ratio was significantly inversely correlated with age.

The current study found that age was correlated with LSM, but Castera et al. (9) reported that the two were not significantly correlated. The current study found that LSM was correlated with BMI, and this finding agrees with the results of Castera et al. (9) and Das et al. (10); in the latter study, the mean value for LSM was higher in obese individuals compared to that in individuals with a normal BMI.

Few studies have discussed the impact of NSBBs on LSM, but Razavi et al. (2) studied the correlation between HVPG and LSM before and after NSBB administration, and they concluded that the two were more strongly and directly correlated post-treatment than pre-treatment.

The correlation between LSM and HVPG improved in hemodynamic responders to NSBBs $(R=0.864)$ but not in non-responders $(R=0.535)$, whereas changes in LSM, heart rate, and mean arterial pressure were similar in both groups (11).

Pre-treatment values for LSM at a cutoff point of 18 $\mathrm{kPa}$ had a sensitivity of $82.4 \%$ and a specificity of $82.6 \%$ at detecting significant EVs ( $\geq$ grade II). Accordingly, LSM is an exceptional test with which to predict the presence of significant EVs. However, other studies used different cutoff values for large EVs. A study by Sporea et al. (12) reported that a cutoff value of $24.8 \mathrm{kPa}$ had a sensitivity of $81 \%$ and a specificity of $80.7 \%$, but that study was a retrospective study, and it did not record LSM in real time or whether patients were given NSBBs or not.

Kazmi et al. (13) conducted a study using cutoff value less than $19 \mathrm{kPa}$ and found that this value was highly predictive for the absence of grade II EVs (sensitivity of $84 \%$, specificity of $78 \%$, PPV of $47 \%$, and NPV of 93\%). Francesco et al. (14) found that LSM at a cutoff value of $17.6 \mathrm{kPa}$ had a sensitivity of $90 \%$, a specificity of $86 \%$, and an NPV of $66 \%$ at predicting EVs. Results of both of those studies agreed with the current results. These variations in cutoff values for LSM to detect or rule out EVs may be related to many factors that include the type of patient, sample size, and different etiologies of cirrhosis.

The current study found that LSM was significantly correlated with the Child-Pugh grade during both preand post-treatment assessments. This finding agreed with the results of Razavi et al. (2), Castera et al. (9), and Foucher et al. (15), who all reported that LSM was correlated with the Child-Pugh score as well as with the stage of fibrosis and EV grade.

The mean PVD decreased significantly after 3 months of therapy with propranolol. With PVD as a noninvasive predictor for EVs, PVD of $12.5 \mathrm{~mm}$ pre-treatment can be used as a cutoff point to predict significant EV with a sensitivity of $82 \%$ and a specificity of $47.8 \%$. Schepis et al. (16) found that PVD of $13 \mathrm{~mm}$ is a cutoff value for the presence of EVs.

The $\mathrm{PC} / \mathrm{SD}$ ratio is considered to be another noninvasive marker for the presence of EVs. In the current study, a PC/SD ratio at a cutoff of 880 had a sensitivity of $94.1 \%$ and a specificity of $69.6 \%$ at predicting significant EVs. Baig et al., (17) found that a $\mathrm{PC} / \mathrm{SD}$ ratio at a cutoff point of 890 was a predictor of EVs with a PPV of $95.5 \%$ and NPV of $95.1 \%$, while Giannini et al. (18) found that a PC/SD ratio at a cutoff point of 909 predicted EVs with a sensitivity of $100 \%$ and a specificity of $71 \%$. The mean value for SD did not change significantly after treatment with NSBBs.

One limitation of the current study was that it did not measure HVPG since doing so would be invasive. Instead, this study evaluated the grade of EVs, which are a common complication of a significant increase in HVPG.

In conclusion, LSM is a noninvasive, reproducible, and rapid method with which to evaluate liver cirrhosis and the stage of fibrosis and with which to evaluate the grade of either small or significant EVs. NSBB therapy can effectively decrease PVD and may consequently improve the EV grade with no significant impact on LSM in patients with liver cirrhosis.

\section{References}

1. Groszmann RJ, Garcia-Tsao G, Bosch J, Grace ND, Burroughs AK, Planas R, Escorsell A, Garcia-Pagan JC, Patch D, Matloff DS, Gao H, Makuch R; Portal Hypertension Collaborative Group. Beta-blockers to prevent gastroesophageal varices in patients with cirrhosis. N Engl J Med. 2005; 353:2254-2261.

2. Razavi H, Waked I, Sarrazin S, et al. The present and future disease burden of hepatitis $\mathrm{C}$ virus (HCV) infection with today's treatment paradigm. J Viral Hepat. 2014; 21 (Suppl 1):34-59. 
3. De Franchis R. Revising consensus in portal hypertension: Report of the Baveno V Consensus Workshop on Methodology of Diagnosis and Therapy in Portal Hypertension. J Hepatol. 2010; 53:762-768.

4. D'Amico G, Garcia-Tsao G, Pagliaro L. Natural history and prognostic indicators of survival in cirrhosis: A systematic review of 118 studies. J Hepatol. 2006; 44:217-231.

5. Berzigotti A, Gilabert R, Abraldes JG, Nicolau C, Bru C, Bosch J, García-Pagan JC. Noninvasive prediction of clinically significant portal hypertension and esophageal varices in patients with compensated liver cirrhosis. Am J Gastroenterol. 2008; 103:1159-1167.

6. Blasco-Algora S, Masegosa-Ataz J, Alonso S, Gutiérrez ML, Fernández-Rodriguez C. Non-selective $\beta$-blockers in advanced cirrhosis: A critical review of the effects on overall survival and renal function. BMJ Open Gastroenterol. 2016; 3:e00104.

7. Sandrin L, Fourquet B, Hasquenoph JM, Yon S, Fournier C, Mal F, Christidis C, Ziol M, Poulet B, Kazemi F, Beaugrand M, Palau R. Transient elastography: A new noninvasive method for assessment of hepatic fibrosis. Ultrasound Med Biol. 2003; 29:1705-1713.

8. Garcia-Tsao G. The use of nonselective beta blockers for treatment of portal hypertension. Gastroenterol Hepatol. 2017; 13:617-619.

9. Castera L, Forns X, Alberti A. Noninvasive evaluation of liver fibrosis using transient elastography. J Hepatol. 2008; 48:835-847.

10. Das K, Sarkar R, Ahmed S, Mridha AR, Mukherjee PS, Das K, Dhali GK, Santra A, Chowdhury A. Normal liver stiffness measure (LSM) values are higher in both lean and obese individuals: A population-based study from a developing country. Hepatology. 2016; 55:584-593.

11. Reiberger T, Ferlitsch A, Payer BA, Pinter M, Homoncik M, Peck-Radosavljevic M; Vienna Hepatic Hemodynamic Lab. Non-selective $\beta$-blockers improve the correlation of liver stiffness and portal pressure in advanced cirrhosis. J Gastroenterol. 2012; 47:561-568.
12. Sporea I, Raţiu I, Bota S, Sirli R, Jurchiş A. Are different cut-off values of liver stiffness assessed by transient elastography according to the etiology of liver cirrhosis for predicting significant esophageal varices. Med Ultrason. 2013; 15:111-115.

13. Kazemi F, Kettaneh A, N'kontchou G, Pinto E, GanneCarrie N, Trinchet JC, Beaugrand M. Liver stiffness measurement selects patients with cirrhosis at risk of bearing large esophageal varices. J Hepatol. 2006; 45:230-235

14. Vizzutti F, Arena U, Romanelli RG, Rega L, Foschi M, Colagrande S, Petrarca A, Moscarella S, Belli G, Zignego AL, Marra F, Laffi G, Pinzani M. Liver stiffness measurement predicts severe portal hypertension in patients with HCV-related cirrhosis. Hepatology. 2007; 45:1291-1297.

15. Foucher J, Chanteloup E, Vergniol J, Castéra L, Le Bail B, Adhoute X, Bertet J, Couzigou P, de Lédinghen V. Diagnosis of liver cirrhosis by transient elastography (Fibroscan): A prospective study. Gut. 2006; 55:403408.

16. Schepis $\mathrm{F}$, Cammà $\mathrm{C}$, Niceforo $\mathrm{D}$, Magnano $\mathrm{A}$, Pallio S, Cinquegrani M, D'amico G, Pasta L, Craxì A, Saitta A, Raimondo G. Which patients with cirrhosis should undergo endoscopic screening for esophageal varices detection? Hepatology. 2001; 33:333-338.

17. Baig WW, Nagaraja MV, Varma M, Prabhu R. Platelet count to spleen diameter ratio for the diagnosis of esophageal varices: Is it feasible? Can J Gastroenterol. 2008; 22:825-828.

18. Giannini E, Botta F, Borro P, Risso D, Romagnoli P, Fasoli A, Mele MR, Testa E, Mansi C, Savarino V, Testa R. Platelet count/spleen diameter ratio: Proposal and validation of a non-invasive parameter to predict the presence of esophageal varices in patients with liver cirrhosis. Gut. 2003; 52:1200-1205.

(Received February 3, 2019; Revised April 25, 2019; Accepted April 29, 2019) 